\title{
RNA activation in RCC: p21 and miR-1236 are a promising pair
}

Combined low expression of cyclindependent kinase inhibitor 1 (p21) and microRNA 1236 (miR-1236) is an independent prognostic factor of poor survival in patients with renal cell carcinoma (RCC), according to a new study in Urologic Oncology. Overexpression of miR-1236 activated p21 expression and inhibited cell proliferation in vitro, pointing towards a role of this pathway in RCC progression and a potential therapeutic strategy.

RNA activation (RNAa) is an emerging mechanism of gene expression regulation. In contrast to RNA interference, some promoter-targeted microRNAs and double-stranded RNAs have been found to increase gene transcription, possibly involving epigenetic mechanisms.

In their study, Chenghe Wang and colleagues from the Huazhong University of Science and Technology in Wuhan, China, first investigated the relevance of p21 and miR-1236 levels to RCC growth. In vitro, they found that a set of RCC cell lines expressed significantly lower levels of these two markers in comparison with primary normal human kidney cells. Investigation of p21 and miR-1236 expression levels in tumour and adjacent normal tissue samples from 45 patients with RCC revealed that their average expression levels were also significantly reduced in the malignant tissue. Levels of both markers were correlated with tumour stage, grade and lymph node involvement. Using multivariate analysis, the team demonstrated that both low expression of p21 alone $(P=0.04)$ as well as combined low p21 and miR-1236 expression $(P=0.01)$ were associated with poor overall survival in patients with RCC.

\section{Overexpression of miR-1236 activated p21 expression and inhibited cell proliferation... 77}

Exploring the relationship between the two markers, the team found that p21 could be a target of miR-1236. In RCC cell lines, overexpression of the microRNA resulted in increased p21 expression, probably via targeting of the $\mathrm{p} 21$ promoter. Importantly, miR-1236 overexpression inhibited cell proliferation and this effect was lost when p21 expression was knocked down using siRNA. In addition, the expression levels of mRNAs and proteins downstream of p21 (cyclin D1 and cyclin-dependent kinases 4 and 6) were reduced after miR-1236 overexpression and this effect was also lost upon p21 knockdown. These data point towards a direct link between miR-1236 and p21 and a central role of p21 in miR-1236dependent regulation of proliferation in the investigated RCC cell lines.

The study provides further insights into the largely unexplored field of RNAa and highlights p21 activation via miR-1236 as a potential novel therapeutic strategy.

\section{Clemens Thoma}

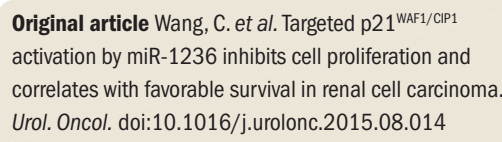

